\title{
Receptores de trasplante renal de donantes en asistolia y muerte cerebral: diferencias en los cuidados enfermeros
}

\author{
$M^{a}$ Concepción García Viana ${ }^{1}$ - Silvia Moral Sánchez ${ }^{1}$ - Raquel Rodríguez Marcos ${ }^{1}$ - Teresa Lope \\ Andrea $^{2}$ - Natalia Ridao Cano ${ }^{3}$
}

\author{
${ }^{1}$ Enfermera \\ ${ }^{2}$ Supervisora \\ ${ }^{3}$ Nefróloga \\ Servicio de Nefrología. Hospital Clínico San Carlos. Madrid
}

\section{Resumen}

Hoy en día es indiscutible que el trasplante renal es el tratamiento sustitutivo de elección para pacientes con enfermedad renal crónica. En nuestro hospital realizamos fundamentalmente dos tipos de trasplante renal, el clásico donante en muerte cerebral y el donante en asistolia. El objetivo de nuestro estudio fue evaluar si existían diferencias en las cargas de trabajo del personal de enfermería dependiendo de un tipo de trasplante u otro.

Se trata de un estudio transversal con 73 pacientes que recibieron un trasplante renal durante el año 2010, 18 procedentes de donante en muerte cerebral y 55 en asistolia. Los trasplante renales de donante en asistolia presentaron mayor incidencia de fallo primario del injerto ( $15 \%$ vs $1,4 \%$ ) así como mayor retraso de la función renal (18 días vs 0 días). Debido a esto, nuestra labor asistencial se vio incrementada en este tipo de trasplante por precisar un mayor número de biopsias renales que implican unos cuidados de preparación y de vigilancia de sus complicaciones, la necesidad de más sesiones de hemodiálisis y la administración de un

Correspondencia:

Raquel Rodríguez Marcos

C/Marroquina, 2, $8^{\circ} \mathrm{F}, 28030$, Madrid

E-mail: raquelrma@yahoo.es mayor número de dosis de timoglobulina. No se objetivaron diferencias significativas en la incidencia de infecciones entre ambos tipo de trasplante, así como en la estancia media hospitalaria, aunque esto último podría estar influido por el tamaño de la muestra.

A pesar de las complicaciones mencionadas, la función renal a medio plazo de ambos tipos de trasplante fue similar, lo que alienta la utilización de los donantes en asistolia.

\section{PALABRAS CLAVE:}

- TRASPLANTE RENAL

- DONANTE EN ASISTOLIA

- DONANTE EN MUERTE CEREBRAL

- CUIDADOS ENFERMEROS

- FALLO PRIMARIO DE INJERTO

- RETRASO DE LA FUNCIÓN RENAL

Recipients of kidney transplants from donors in asystole and brain death: differences in nursing care

\section{Abstract}

Nowadays it is indisputable that kidney transplant is the preferred replacement therapy for patients 
with chronic kidney disease. In our hospital we carry out basically two types of kidney transplants, the classic brain dead donor and the asystolic donor. The aim of our study was to evaluate whether there were differences in the workload of nursing staff depending on the type of transplant.

This is a transversal study of 73 patients who received a kidney transplant in 2010, 18 of which were from a brain dead donor and 55 from an asystolic donor. The kidney transplants from asystolic donors showed a higher incident of primary graft failure (15\% v. $1.4 \%$ ) and a greater delay in kidney function (18 days v. 0 days). Because of this, our care task was increased in this type of transplant as a greater number of kidney biopsies were required which involve care in preparation and supervision of complications, the need for more haemodialysis session and the administration of a more doses of thymoglobulin. No significant differences were identified in the incidence of infections between the two types of transplant, or in the average hospital stay although the latter could be influenced by the size of the sample.

Despite the complications mentioned above, kidney function in the medium term in both types of transplant was similar, which encourages the use of asystolic donors.

\section{KEY WORDS:}

- KIDNEY TRANSPLANT

- ASYSTOLIC DONOR

- BRAIN DEAD DONOR

- NURSING CARE

- PRIMARY GRAFT FAILURE

- DELAY IN KIDNEY FUNCTION

\section{Introducción}

Hoy en día es indiscutible que el trasplante renal (TxR) es el tratamiento sustitutivo de elección para pacientes con enfermedad renal crónica (ERC). Porque mejora la morbimortalidad, además de suponer un menor coste económico a medio plazoํ.

La incidencia de los pacientes con ERC que precisan tratamiento renal sustitutivo ha aumentado casi al doble en la última década, mientras que el número de potenciales donantes no ha demostrado incremento sustancial, razón por la cual se han ampliado los criterios de los donantes como por ejemplo el donante añoso, el donante en asistolia y se está promoviendo el donante vivo ${ }^{1,2}$.

Los riñones procedentes de donantes en asistolia son aquellos que se extraen tras una parada cardiorespiratoria irreversible intra o extrahospitalaria (siendo esta última la más frecuente) y que deben cumplir unos estrictos criterios, sobre todo con respecto a los tiempos de isquemia ${ }^{3}$.

El hospital Clínico San Carlos comenzó su programa de trasplantes renales en 1971 y hasta diciembre de 2010 se han realizado 1649 trasplantes. En 1989 se puso en marcha el protocolo de trasplante de donante en asistolia pionero en España.

La cuestión clave en los donantes a corazón parado con respecto a los donantes de muerte cerebral, es la mayor incidencia de fallo primario del injerto ${ }^{3,4}$ (injerto nunca funcionante) y el retraso en la función renal relacionado fundamentalmente con el tiempo de isquemia caliente ${ }^{5}$. Esto puede conllevar diferencias en los cuidados enfermeros a administrar en el periodo postrasplante ${ }^{6}$.

El objetivo de nuestro estudio fue evaluar las diferencias en la carga de trabajo del personal de enfermería dependiendo del tipo de donante.

\section{Material y métodos}

Se trata de un estudio transversal realizado entre enero y diciembre de 2010. La población a estudio fueron 73 pacientes con ERC y el criterio de inclusión fue haber recibido trasplante renal durante este periodo.

Las variables recogidas para realizar el estudio fueron:

Cuantitativas: edad del receptor, edad del donante, número de hemodiálisis durante el ingreso, número de biopsias, días de permanencia de catéter "Doble 
$\mathrm{J}^{\prime \prime}$, días de permanencia de sonda vesical, días de ingreso, creatinina al alta, creatinina en consulta y días de retraso de la función renal.

Cualitativas: tipo de trasplante (asistolia/muerte cerebral), trasplante funcionante/fallido, sexo, situación previa, administración de timoglobulina pretrasplante, administración de timoglobulina postrasplante, incidencia de infección del tracto urinario, infección respiratoria, infección de vía central e infección de herida quirúrgica y desarrollo de diabetes mellitus (DM).

Las variables cualitativas se presentan con su distribución de frecuencias. Las variables cuantitativas se resumen con su media y desviación estándar $(D E)$ en caso de que se distribuyan de forma normal, o con su mediana y rango intercuartílico (RIQ) en caso de no seguir una distribución normal. En todos los casos se comprobó la distribución de la variable frente a los modelos teóricos. Se evaluó la asociación entre variables cualitativas con el test de $X^{2}$ o prueba exacta de Fisher, en el caso de que más de un $25 \%$ de los esperados fueran menores de 5 .

Se analizó el comportamiento de las variables cuantitativas por cada una de los grupos estudiados mediante el test de la $t$ de Student o con test no paramétricos (test $\mathrm{U}$ de Mann-Whitney o test de la mediana en tamaños muestrales pequeños) En todos los contrastes de hipótesis se rechazó la hipótesis nula con un error de tipo I menor a 0,05. El paquete informático utilizado para el análisis fue SPSS para Windows versión 11.0 y la recogida de datos fue a través de la revisión de historias clínicas y del programa informático "Gacela".

El protocolo de cuidados de los candidatos es igual para los dos tipos de TxR: acogida del paciente, realización de pruebas preoperatorias, manejo de muestras, realización de sesión de hemodiálisis o intercambios de diálisis peritoneal, preparación del paciente y administración de medicación antes de la cirugía (fundamentalmente inmunosupresión y corticoides). Tras la intervención y una corta estancia en UCI (aproximadamente 24 horas) el paciente es recibido en la unidad de aislamiento de TxR donde se continúan con los cuidados postoperatorios.

\section{Resultados}

Para el análisis de nuestro estudio debemos considerar que el tamaño de la muestra es relativamente pequeño y que las características clínicas que pueden influir en la evolución funcional de los injertos son muy diversas, a saber, causa de muerte del donante, tipo de parada cardiaca, técnica de preservación, edad del donante y del receptor, trasplantes previos, diferentes protocolos de inmunosupresión, etc ${ }^{7}$. Todo esto hace que los resultados deban ser interpretados con cautela.

La administración de Timoglobulina en el período Pretx se dio en el $80,8 \%$, de los cuales el $100 \%$ fue de Asistolia y el $22,2 \%$ de MC (Receptores con Tx previos o de donantes añosos).

Se administró Timoglobulina en el período Postx en el $89 \%$ de los casos: $69,9 \%$ de Asistolia y el $19,2 \%$ de MC.

Existe diferencia significativa en el número de HD que recibieron los receptores de riñones prodecende de donantes en Asistolia [3 RIQ (1-5)] frente a los de MC [0 RIQ (0-2)] ( $P=0,009 \mathrm{U}$ de Mann-Whitney), así como en el número de biopsias renales realizadas en el periodo postTx [1 (RIQ 0-3) en los receptores de asistolia y de 0 (RIQ de 0-3) en los de muerte cerebral $(P=0,014$. Razón de verosilimitudes $)]$.

El resto de resultados se muestran en gráficos y tablas representados.

\begin{tabular}{|l|c|c|c|c|} 
& Total & Asistolia & Muerte Cerebral & P \\
\hline I. Urinaria & $14(19,2 \%)$ & $11(15,1 \%)$ & $3(4,1 \%)$ & $1,000^{*}$ \\
\hline I. Respiratoria & $8(11 \%)$ & $7(12,7 \%)$ & $1(1,4 \%)$ & $0,670^{*}$ \\
\hline I. Vía Central & $2(2,7 \%)$ & $1(1,4 \%)$ & $1(1,4 \%)$ & $0,435^{*}$ \\
\hline I. Herida quirúrgica & $4(5,5 \%)$ & $0(0 \%)$ & $4(5,5 \%)$ & $0,566^{*}$ \\
\hline (*Estadístico de Fisher) & & & & \\
\hline
\end{tabular}

Tabla 1. Desarrollo de Infecciones 


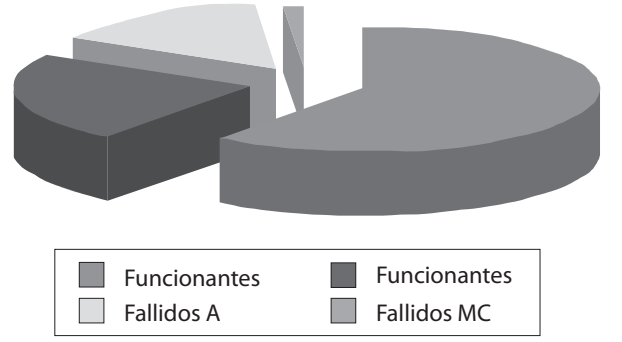

Gráfico 1. Relación de injertos funcionantes y fallidos

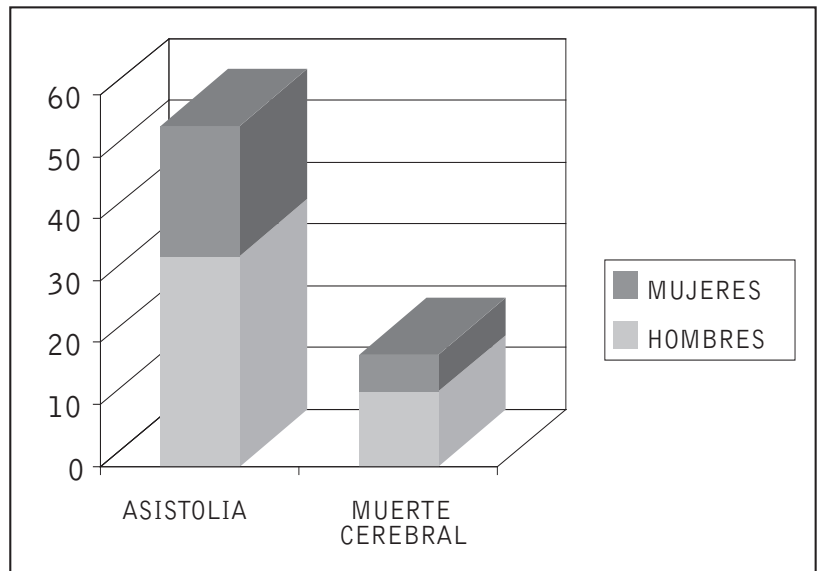

Gráfico 2. Porcentaje de trasplantes entre hombres y mujeres

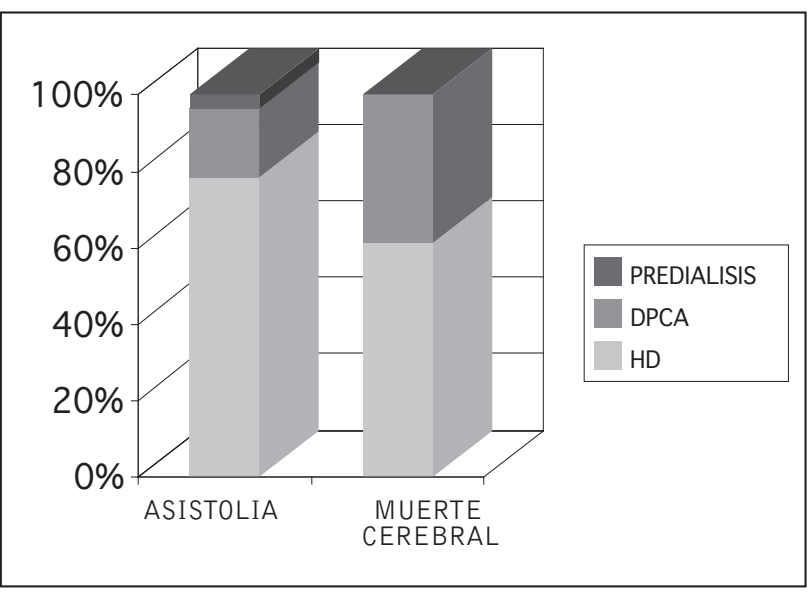

Gráfico 3. Situación basal previa de los pacientes trasplantados

\section{Discusión}

Coincidiendo con la literatura ${ }^{3,4}$, nuestro estudio muestra que los TxR procedentes de donante en asistolia presentan mayor incidencia de fallo primario del injerto (injerto nunca funcionante) y mayor retraso en la función renal con respecto a los TxR de donantes en muerte cerebral. Paralelamente, hemos observado que la labor asistencial del personal de enfermería se ve incrementada en los TxR en asistolia por las siguientes circunstancias:

\begin{tabular}{|l|c|c|c|c|}
\hline & Total & Asistolia & Muerte Cerebral & P \\
\hline Desarrollo DM & $33(50,8 \%)$ & $25(38,5 \%)$ & $8(12,3 \%)$ & \\
\hline No desarrollo & $32(49,2 \%)$ & $24(36,9 \%)$ & $8(12,3 \%)$ & $0,943 * * *$ \\
\hline$(* * *$ Chi-cuadrado de Pearson) & & & \\
\hline
\end{tabular}

Tabla 2. Desarrollo de Diabetes Mellitus

\begin{tabular}{|c|c|c|c|c|}
\hline & Total & Asistolia & Muerte cerebral & $\mathbf{P}$ \\
\hline Días de permanencia de "Doble J" & 12 (RIQ 10-14) & $12,22 \pm 4,077$ & $13,56 \pm 6,706$ & $0,312^{*}$ \\
\hline Días de permanencia de sondaje vesical & 12 (RIQ 10-14) & 12 (RIQ 10-14) & 12 (RIQ 11-15) & 0,396 ** \\
\hline Días de retraso de la función renal & 15,50 (RIQ 6,25-22) & 18 (RIQ 15-23) & 0 (RIQ 0-7) & 0,001 ** \\
\hline Días de ingreso & $24,49 \pm 9,94$ & $24,67 \pm 9,53$ & $23,88 \pm 11,45$ & $0,349 * *$ \\
\hline Creatinina al alta & $3,96 \pm 2,587$ & $5,082 \pm 2,514$ & $2,056 \pm 1,514$ & $0,001^{*}$ \\
\hline Creatinina en consulta & $1,62 \pm 0,432$ & $1,665 \pm 0,414$ & $1,500 \pm 0,466$ & $0,185^{*}$ \\
\hline
\end{tabular}

Tabla 3. Relación de Variables Cuantitativas 
1) la necesidad de administrar un mayor número de dosis de timoglobulina intravenosa para evitar el uso inicial de anticalcineurínicos (tacrolimus) disminuyendo así su efecto nefrotóxico ${ }^{8}$. Esto conlleva el cuidado y mantenimiento durante más tiempo de una vía central o periférica adecuada.

2) la realización de mayor número de biopsias renales, con sus cuidados correspondientes, y de sesiones de hemodiálisis debido al retraso en la función renal del TxR en asistolia.

3) la mayor vigilancia por parte de enfermería de cualquier signo de alarma (ejemplo: dolor en el injerto, hematuria, hipotensión, etc) que haga sospechar un fallo primario del injerto que conlleva inexorablemente a una cirugía urgente de extracción del TxR.

A pesar de estas diferencias mencionadas, no hubo más infecciones en un tipo TxR u otro, y éstas tuvieron una incidencia muy baja. Esto podría estar relacionado con la existencia en nuestro hospital de una unidad especial de aislamiento y un entrenamiento especifico del personal de enfermería siguiendo las estrictas medidas de asepsia.

Otro aspecto importante es la estancia media hospitalaria. En términos generales, una función renal óptima temprana implicaría una menor estancia hospitalaria. Sin embargo, en nuestro estudio no observamos diferencias significativas en el tiempo de estancia media hospitalaria entre un tipo y otro de $T x R$, a pesar de las diferencias claras en la función inmediata del injerto. Esto podría estar influenciado por ciertos casos de ingresos prolongados que pueden afectar a los resultados dado el tamaño relativamente pequeño de la muestra.

Finalmente cabe destacar que al igual que en otras publicaciones ${ }^{9,10}$ nuestros resultados indican que a pesar de cifras de creatinina sérica más elevadas inicialmente en los receptores de asistolia, el funcionamiento a medio plazo es superponible a los obtenidos de donantes en muerte encefálica.

Podemos concluir que los pacientes que reciben un TxR de donante en asistolia, comparado con la muerte cerebral, requieren mayores cuidados y vigilancia más intensiva por parte de enfermería pero finalmente la función renal es similar, por lo que debemos estimular este tipo de trasplante.

Recibido: 20 0ctubre 2011

Revisado: 15 Noviembre 2011

Modificado: 29 Diciembre 2011

Aceptado: 18 Enero 2012

\section{Bibliografía}

1. Morales Ruiz E. Trasplante renal anticipado. Guias SEN. Volumen 28. 2008; 3: 123-128.

2. Lopez-Navidad A., Kulisevsky J., Caballero F. El donante de órganos y tejidos. Springer-Verñag Ibérica, Barcelona 1997; 3: 27.

3. Ortega F., Arias M., Campistol J.M., Matesanz R., Morales J.M. Trasplante Renal. Médica Panamericana, 2007; 3: 39.

4. Rampino T, Abelli M, Ticozzelli E, Gregorini M. Nonheart-beating-donor transplant: the first experience in Italy. PMID: 20191461 [PubMed- indexed for Medline] 2010 Jan- Feb; 27(1):56-68 [consultado el 6 de abril de 2011]. Disponible en http://www. ncbi.nlm.nih.gov/pubmed/20191461.

5. Kosieradzki M, Rowinski W. Ischemia/reperfusion injury in kidney transplantation: mechanisms and prevention. PMID: 19100373 [PubMed- indexed for Medline] 2008 Dec; 40(10):32-79-88 [consultado el 6 de abril de 2011]. Disponible en http:// www.ncbi.nlm.nih.gov/pubmed/19100373.

6. Sánchez-Fructuoso AI, Prats D, Torrente J, PérezContin MJ, Fernandez C, Álvarez J, Barrientos A. Renal transplantation from non-heart beating donors: a promising alternative to enlarge the donor pool. J Am Soc Nephrol 2000; 11: 350-358.

7. Kimber RM, Metcalfe MS, White SA, Nicholson $M L$. Use of nos-heart-beating donors in renal transplantation. Postgrad Med J 2001;77:681-685. 
8. Gutiérrez-Solís E, Fernández Juárez G. NefroPlus. Junio 2010. 3(1):16-27 [consultado 1 abril de 2011]. Disponible en http://www.revistanefrologia. com/modules.php? name $=$ articulos $\&$ idarticulo $=104$ $71 \&$ idlangart=ES.

9. Palencia Herrejón E. Trasplante renal de donantes en asistolia. Revista electrónica de medicina inten- siva. Julio 2002. Art 418. Vol $2 n^{\circ} 7$. [consultado 2 abril de 2011]. Disponible en http://remi.uninet. edu/2002/07/REMI0418.htm.

10. Andreu Periz L, Force Sanmartin E. La enfermería y el trasplante de órganos. Médica Panamericana, 2004; 2: 22. 